\title{
The axiomatic method, the order of concepts and the hierarchy of sciences: an introduction
}

\author{
Arianna Betti · Willem R. de Jong • \\ Marije Martijn
}

Received: 25 July 2009 / Accepted: 11 August 2009 / Published online: 6 October 2009

(C) The Author(s) 2009. This article is published with open access at Springerlink.com

This special issue of Synthese 'The Classical Model of Science II: The axiomatic method, the order of concepts and the hierarchy of sciences' follows up on the previous 'The Classical Model of Science I: A millennia-old model of scientific rationality'. Both issues centre on the role, the significance and the impact of the axiomatic ideal of scientific knowledge in the history of philosophy. The first issue focuses on the relation between axiomatics and a number of issues in the development of logic, mathematics, and methodology and philosophy of science in Aristotle, Proclus, the seventeenth century, Kant, Bolzano, Frege and Leśniewski. The papers collected in this second issue on the one hand continue to investigate that relation in Kant and Bolzano, stretching it further on to our days, via mathematicians such as Schröder, Dedekind, and Birkhoff, and on the other hand they extend that investigation to related and current issues concerning the empirical sciences, in a systematic evaluation of modern (formal) axiomatic conceptions of science. The contributions in both issues take their cue from the axiomatic ideal in question as captured in the 'Classical Model (or Ideal) of Science' (de Jong and Betti 2008):

(1) All propositions and all concepts (or terms) of $S$ concern a specific set of objects or are about a certain domain of being $(s)$.

(2a) There are in $S$ a number of so-called fundamental concepts (or terms).

\footnotetext{
A. Betti $(\varangle) \cdot$ W. R. de Jong · M. Martijn

Faculteit der Wijsbegeerte, Vrije Universiteit Amsterdam, De Boelelaan, 11051081 HV Amsterdam, The Netherlands e-mail: a.betti@ph.vu.nl

W. R. de Jong

e-mail: w.r.de_jong@ph.vu.nl

M. Martijn

e-mail: M.Martijn@ph.vu.nl
} 
(2b) All other concepts (or terms) occurring in $S$ are composed of (or are definable from) these fundamental concepts (or terms).

(3a) There are in $S$ a number of so-called fundamental propositions.

(3b) All other propositions of $S$ follow from or are grounded in (or are provable or demonstrable from) these fundamental propositions.

(4) All propositions of $S$ are true.

(5) All propositions of $S$ are universal and necessary in some sense or another.

(6) All propositions of $S$ are known to be true. A non-fundamental proposition is known to be true through its proof in $S$.

(7) All concepts or terms of $S$ are adequately known. A non-fundamental concept is adequately known through its composition (or definition).

These seven conditions systematize desiderata of meaningfulness, economy, definability, ground and consequence, truth, necessity, and knowability of propositions and concepts (or sentences and terms) of a real or proper science. Conditions (1-5) relate primarily to the ontological 'order of things' (ordo essendi); they can be divided into what we call the 'Domain Postulate' (1), the 'Postulate of Order' (2a, 2b; 3a, 3b), the 'Truth Postulate' (4) and the 'Necessity' and the 'Universality Postulate' (5). Conditions (6) and (7) instead, together forming 'the Postulate of (Grounded) Knowledge', regard the epistemological 'order of knowledge' (ordo cognoscendi). It is worth mentioning that what is at issue here is an explanatory ideal of scientific knowledge: what follows from the principles (axioms and definitions) is explained by those principles, which function as grounds. The relation of 'consequence' (or, in all generality, of 'following') ad 3b should be understood accordingly. The Classical Model of Science as set down in conditions (1-7) can be more immediately seen as concerning an organized system of known truths from the point of view of the context of justification; however, it is also relevant to the context of discovery (ars inveniendi/methodus inventionis), insofar as it can be approached as a normative guide for finding scientific truths, for instance as the ideal structure to be aimed at in seeking to establish axioms. Conditions (1-7) also provide a common interpretive grid and terminology, variously taken up by the papers which follow.

Hein van den Berg's 'Kant's conception of proper science' analyses Kant's take on the Postulate of Order and on the Necessity Postulate. The Postulate of Order concerns the systematic ordering of concepts by means of definition (i.e. $2 a, 2 b$ ) and of propositions or judgements by means of relations of grounding (i.e. $3 \mathrm{a}, 3 \mathrm{~b}$ ); the Necessity Postulate, in one construal, concerns the constraint that the judgements of a proper science must be grounded in a priori principles, which is part of (5). Both Postulates underlie Kant's conception of a proper science, which van den Berg analyses as any body of cognition which (i) is organized into a complete systematic whole, (ii) expresses objective ground-consequence relations and (iii) has a priori principles from which the non-fundamental judgements of a science can be proven. On the basis of this analysis van den Berg offers an interpretation of Kant's notion of mathematization, i.e. the claim that any real science should be mathematical, which enables him to explain two things: the specifically foundational role of mathematics with respect to physics and the special status of mathematical natural sciences as the only real natural sciences. 
The epistemic conditions on the knowability of propositions and terms (i.e. 6, 7), and in particular Bolzano's views on the knowability of axioms in the process of discovery, are at the core of Anita Konzelmann's 'Bolzanian knowing: Infallibility, virtue and foundational truth', based on the little studied epistemological sections of Bolzano's Wissenschaftslehre. In contemporary terms Bolzano emerges from Konzelmann's analysis as a virtue epistemologist "in its responsibilist version, which combines reliabilist concerns for epistemic success with internalist concerns for agentive responsibility for knowledge". For Bolzano, knowing that a proposition is an axiom means asserting thus, and truly evaluating one's asserting thus as infallible. The latter requires a (non-assertive) account of the evaluation of an assertion as genuinely immediate, but since there are no structural means to distinguish immediate from mediate assertions, Bolzanian knowledge seems to collapse onto a very high degree of believing. Can we avoid this? Not exactly. Konzelmann argues that our best option is to take Bolzanian knowing to involve the best possible evaluation of an assertion's fallibility while relying on certain salient virtues of the knower, such as trust and prudence.

The context of discovery figures prominently also in 'On the creative role of axiomatics. The discovery of lattices by Schröder, Dedekind, Birkhoff, and others', by Dirk Schlimm, which centres on the Domain Postulate (i.e. condition (1)). Schlimm discusses the independent introduction of lattices by Schröder, Dedekind, Birkhoff and others as examples illustrating three different ways in which axiomatic systems can creatively lead to the discovery of new domains of objects of investigation: by analogy, by abstraction and by modification. In the first case we start with the consideration of a similarity between certain domains and then capture the similarity by setting up a common axiom system; in the second case we select properties of a given domain, then we capture these properties axiomatically and finally we identify new domains as those which also satisfy the axioms in question; in the third case we modify the axiom system directly by adding, deleting or changing one or more axioms and then use the system thus obtained to define a new domain.

The method of abstraction in particular was made possible by the new conception of formal axiomatic systems which emerged at the end of the nineteenth century, in which primitives are conceived as reinterpretable. This new, formal conception of axiomatics is also interestingly connected with the birth of model theory. The model-theoretical approach to axiomatics, in particular the model-theoretical notion of logical consequence-rather than the proof-theoretical one-forms the specific take on (3a, 3b) that Jaakko Hintikka champions in 'What is the axiomatic method?'. An axiomatic system in the modern sense, says Hintikka, is the study of a certain class of structures, i.e. its models. The derivation of theorems from axioms can produce only new 'surface' (or explicit) information, not new 'depth' (or implicit) information. That is, it can only produce information that can be read off a sentence without non-trivial deductive aids. Axioms cannot make theorems any more certain, and in this sense they occupy no epistemologically privileged position. Still, axioms can fulfill an explanatory role with respect to the theorems. A partial measure of this explanatory ability of axioms is their simplicity. Hintikka's proposal may be read as the modern counterpart of the traditional constraint that the relation of consequence between axioms and theorems 
is one in which axioms ground their theorems, as discussed in van den Berg's and Konzelmann's papers on Kant and Bolzano.

Like Hintikka's paper, the last two papers of this issue offer a broad discussion of (formal) axiomatic views of science in general and are model-theoretically oriented; however, they lay more emphasis on (the philosophy of) empirical science. F.A. Muller's 'Reflections on the revolution at Stanford' is a critical discussion of the revolutionary change brought about by Patrick Suppes' view of the nature of scientific theories ('the Model Revolution'). Muller's criticism is related to the Domain and the Truth Postulates (conditions (1) and (4) above). A scientific theory in Suppes' 'Informal Structural View' is a set of set-structures defined in the language of pure set theory. But, Muller counters, a scientific theory is supposed to give us scientific knowledge in the sense of scientifically justified true propositions about concrete actual beings. In a Suppesian scientific theory it is glossed over how to discern which data are relevant for which theory, and there is no place reserved for either beings, language, or the relations between the structures that constitute the theory and the beings the theory is supposed to be about. Muller proposes to solve these problems and to complete 'the Model Revolution' by what he calls 'the Structural View', which involves, in Muller's opinion, a better characterization of what scientists call 'model', that is, a structure meeting a certain set-theoretical predicate (the Suppes-predicate) together with all its formulations. A discussion of problems concerning the currently much debated notion of scientific representation concludes the paper.

In his response to Muller, 'Future development of scientific structures closer to experiments', Patrick Suppes discusses a different direction for future developments of his Informal Structural View, which aims at solving a number of problems concerning the philosophy of science, especially experimental science. Suppes pleads for staying in touch as much as possible with the actual practices of science at the level of measurement, observation and computation, and with how these practices "should be reflected back into the theory when the limitations imposed by errors or environmental variations are taken seriously". Suppes attracts attention to the contrast between experiment-talk and theory-talk; to the non-verbal aspects of experimental practice and the way in which experimental methods have influenced the progress of both natural and mathematical sciences in the past; and to the fact that invariance as sought after in approaches like Muller's tends to be incompatible with computational simplicity, while it is often the latter which is preferred in scientific practices. Suppes touches upon four programmatic issues: first, structures which, albeit still formally defined, match more closely actual methods of measurement, are to be preferred to classical structures with infinite domains closely matching mathematical ones; secondly, axioms of floating-point arithmetic should replace the classical axioms of arithmetic for computational purposes; thirdly, ergodic theory of chaos should be used to get general results about errors; and the fourth final programmatic step is one towards a constructive, non-standard foundation of analysis.

The publication of this second special issue concludes a project on the Classical Model of Science partly funded by the Netherlands Organisation for Scientific Research (project 275-80-001) and by the European Research Council (project 203194). The project involved a conference in Amsterdam which, like this issue, was entitled 'The axiomatic method, the order of concepts and the hierarchy of sci- 
ences', with an open call for papers, where earlier versions of the papers by Anita Konzelmann, Jaakko Hintikka, Dirk Schlimm and F.A. Muller were presented. The paper by Hein van den Berg was written especially to be submitted to this issue. The paper by Patrick Suppes was originally a long reply on Muller's paper in the form of a letter to the author. We invited Patrick Suppes to submit the reply in paper form.

We wish to thank Stefan Roski and Jan Willem Wieland for meticulous editorial assistance on the final versions, and the anonymous referees for their precious time and kind help with this project. Finally, thanks to Jan Woleński, and to the editors of Synthese, especially John Symons, for having believed in this project and for supporting it by hosting both special issues in the journal series.

Open Access This article is distributed under the terms of the Creative Commons Attribution Noncommercial License which permits any noncommercial use, distribution, and reproduction in any medium, provided the original author(s) and source are credited.

\section{Reference}

de Jong, W. R., \& Betti, A. (2008). The classical model of science: A millennia-old model of scientific rationality. Synthese. Online first at http://www.springerlink.com/content/w5340m712258304m. doi:10.1007/s11229-008-9416-5. 